\title{
Management of acute paracetamol poisoning
}

\author{
Matthew SH Tsui *, FRCP (Edin), FHKAM (Emergency Medicine) \\ Department of Accident and Emergency, Queen Mary Hospital, Pokfulam, Hong Kong
}

Hong Kong Med J 2015;21:388

*tsuish@ha.org.hk

DOI: $10.12809 / \mathrm{hkmj} 154680$

Since 2010, paracetamol has been the most common agent used in Hong Kong for deliberate self-harm by overdose and poisoning. ${ }^{1}$ It is readily available over-the-counter and is commonly prescribed by doctors. There are more than 900 registered pharmaceuticals that contain paracetamol in Hong Kong. Paracetamol overdose can result in delayed, sometimes life-threatening, liver injury and dosedependent damage. $\mathrm{N}$-acetylcysteine (NAC) is well known to be an effective antidote that can prevent liver injury if administered in time. The decision to give NAC can be facilitated by plotting the serum paracetamol concentration against time since ingestion on the Rumack-Matthew nomogram. ${ }^{2}$ Serum concentration above the treatment line on the nomogram indicates the need for NAC therapy.

There are three treatment lines on the Rumack-Matthew nomogram: 100-treatment line, 150-treatment line, and 200-treatment line. Currently, the 150-treatment line is commonly used in most parts of the world including the US, Australia, and New Zealand. The 150-treatment line is parallel to the original 200-treatment line but has been arbitrarily lowered by $25 \%$ to improve sensitivity. The Hong Kong Poison Information Centre also recommends the 150-treatment line and most clinicians in Hong Kong follow this recommendation. In the UK, the original 200-treatment line was used for normal-risk patients and the 100-treatment line reserved for high-risk patients. Over the years, cases of liver failure accumulated when patients were treated according to the 200-treatment line. Thus in 2012 the health department in the UK decided to abandon the two-level approach and apply one treatment line of $100 \mathrm{mg} / \mathrm{L}$ for all patients. ${ }^{3}$

In the article written by Chan et $\mathrm{al}^{4}$ the 150-treatment line has been evaluated and identified a failure rate of $0.45 \%$. All four index patients developed chemical hepatitis that responded to supportive treatment. The incidence of 150-treatment line failures in the US has been reported as $1 \%$ to $3 \%$ and thought to be predominantly due to inaccurate ingestion history. ${ }^{5}$ Looking closely at the four cases presented in Chan et al's study, ${ }^{4}$ two of them presented late, and in most cases there was an apparent discrepancy between the dose taken and the achieved paracetamol level. Similar to the US experience, an inaccurate ingestion history might explain treatment-line failure for some of these cases. Further evidence from more robust studies is needed before a recommendation can be made to lower the treatment threshold to the UK standard.
Obtaining an accurate history from patients who deliberately self-harm is known to be difficult. Patients may be unwilling or unable to provide accurate information to the clinician. According to the author's own experience in managing such patients, history taking must be done tactfully and sometimes repeatedly from different sources of information. An astute physician should make the decision to give NAC after analysing all the available evidence including the best-gathered history, the clinical presentation, and the remaining treatmenttime window, together with the serum paracetamol level. Laboratory tests may help but can never replace clinical skill, clinical judgement, and experience in patient management.

Previously, the responsibility for managing such time-critical overdosed patients was often borne by interns and junior residents. The quality of care provided may not have been optimal. Over the past 10 years emergency physicians and trainees have received intensive training in the management of toxicology cases based on updated evidence and standards. In addition, groups of interested emergency physicians have formed toxicology teams to oversee and support the management of poisoning patients in individual hospitals. This model of care improves patient outcome and shortens the length of stay for medical treatment. ${ }^{6,7}$ Such improvements might explain the observed good outcome for Chan et al's cohort ${ }^{4}$ of patients with paracetamol overdose.

\section{References}

1. Chan YC, Tse ML, Lau FL. Hong Kong Poison Information Centre: Annual Report 2013. Hong Kong J Emerg Med 2014;21:249-59.

2. Rumack BH, Matthew H. Acetaminophen poisoning and toxicity. Pediatrics 1975;55:871-6.

3. Paracetamol overdose: new guidance on use of intravenous acetylcysteine. Commission of Human Medicine, United Kingdom. Available from: http://www.mhra.gov.uk/home/ groups/pl-p/documents/drugsafetymessage/con 178654 . pdf. Accessed Aug 2015.

4. Chan ST, Chan CK, Tse ML. Paracetamol overdose in Hong Kong: is the 150-treatment line good enough to cover patients with paracetamol-induced liver injury? Hong Kong Med J 2015;21:389-93.

5. Rumack BH. Acetaminophen hepatotoxicity: the first 35 years. J Toxicol Clin Toxicol 2002;40:3-20.

6. Chung AH, Tsui SH, Tong HK. The impact of an emergency department toxicology team in the management of acute intoxication. Hong Kong J Emerg Med 2007;14:134-43.

7. Ko S, Chan HY, Ng F. The impact of Emergency Medicine Ward in acute intoxication management. Hong Kong J Emerg Med 2010;17:323-31. 